\section{Protecting mitochondrial bioenergetic function}

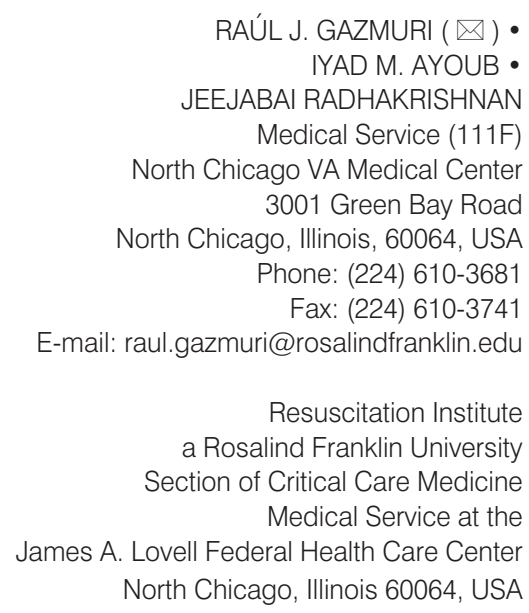

RAÚL J. GAZMURI $(\bowtie) \bullet$ IYAD M. AYOUB • JEEJABAI RADHAKRISHNAN Medical Service (111F) North Chicago VA Medical Center 3001 Green Bay Road North Chicago, Illinois, 60064, USA Phone: (224) 610-3681 Fax: (224) 610-3741

E-mail: raul.gazmuri@rosalindfranklin.edu

Resuscitation Institute a Rosalind Franklin University Section of Critical Care Medicine Medical Service at the James A. Lovell Federal Health Care Center North Chicago, Illinois 60064, USA

\begin{abstract}
Reversal of cardiac arrest requires reestablishment of aerobic metabolism by reperfusion with oxygenated blood of tissues that have been ischemic for variables periods of time. However, reperfusion concomitantly activates a myriad of pathogenic mechanisms causing what is known as "reperfusion injury." At the center of reperfusion injury are mitochondria, playing a critical role as effectors and targets of injury. Mitochondrial injury compromises oxidative phosphorylation and the ability to regenerate Adenosine-5'-triphosphate (ATP); i.e., bioenergetic function. Thus targeting mitochondria to protect bioenergetic function may represent a novel concept in resuscitation with the potential for altering clinical practice. We have identified sodium-hydrogen exchanger isoform-1 (NHE)-1 inhibition and erythropoietin as attractive candidate drugs for this purpose and demonstrated corresponding functional and clinical benefits. Further work on the subject may pave the way for further scientific discover focused on greater understating of underlying cell mechanisms, identification of additional and perhaps more potent strategies, and develop means for effective drug delivery.
\end{abstract}

Keywords: cardiac arrest, reperfusion injury, mitochondria, bioenergetic function, left ventricular myocardial distensibility, sodium-hydrogen exchanger isoform-1 (NHE-1) inhibitors, erythropoietin

\section{Introduction}

The working heart is a highly metabolic organ that under normal resting conditions extracts nearly $70 \%$ of the oxygen supplied by the coronary circulation $(1,2)$ representing close to $10 \%$ of the total body oxygen consumption. However, the heart has minimal capability for extracting additional oxygen such that increases in metabolic demands can only be met by autoregulatory increases in coronary blood flow through vasodilation of the coronary circuit. (3) Con- sequently, a severe energy imbalance develops when cardiac arrest occurs and coronary blood flow ceases. The severe energy imbalance continues during the ensuing resuscitation effort when current closed-chest resuscitation techniques are used because of the very limited capability for generating systemic and coronary blood flow. (4) Moreover, with reperfusion during resuscitation, multiple pathogenic mechanisms - collectively known as "reperfusion injury" - are activated and further contribute to myocardial injury. Main contributors to reperfusion injury are mitochondrial $\mathrm{Ca}^{2+}$ overload $(5,6)$ and generation of reactive oxygen species (ROS). (7)

Various functional myocardial abnormalities develop consequent to ischemia and reperfusion during cardiac arrest and resuscitation that exert effects detrimental to cardiac resuscitation. These abnormalities can be grouped into those that manifest during the resuscitation effort and those that manifest after the return of spontaneous circulation. The former include reductions in left ventricular myocardial distensibility and increased resistance to electrical defibrillation; the latter include reperfusion arrhythmias and post-resuscitation myocardial dysfunction.

Work from our laboratory supports the concept that interventions able to protect mitochondrial bioenergetic function can prevent reductions in left ventricular myocardial distensibility during cardiopulmonary resuscitation (CPR), facilitate return of spontaneous circu- 
Iation, ameliorate post-resuscitation myocardial dysfunction and ultimately improve resuscitation and survival with intact neurological function.

\section{Left Ventricular Myocardial Distensibility}

In this article we summarize evidence in support that preservation of mitochondrial bioenergetic function can prevent reductions in left ventricular myocardial distensibility and facilitate successful resuscitation and survival.

Salient features: Studies in various animal models of ventricular fibrillation (VF) and resuscitation have shown progressive thickening of left ventricular wall accompanied by parallel reductions in left ventricular cavity without changes in intracavitary pressures during the resuscitation effort. $(8,9)$ Reductions in left ventricular myocardial distensibility is likely a manifestation of myocardial reperfusion injury that presents with the following salient features: i) onset and progression during chest compression, $(8,9)$ ii) modest ATP depletion (10) iii) mechanism likely to represent energy deficit compounded by cytosolic and mitochondrial $\mathrm{Ca}^{2}+$ overload precluding complete relaxation of individual cardiomyocytes, iv) decreased distensibility leading to diastolic dysfunction upon return of spontaneous circulation, (11) and v) largely a reversible phenomenom. (12)

Hemodynamic consequences: As blood returns to the heart during the relaxation phase of chest compression, distensible ventricles are important to properly accommodate the returning blood and establish an adequate preload for the subsequent compression. The larger the distensibility, the larger the preload, and the larger the amount of blood that can be ejected by chest compression. This mechanism is akin to the Frank-Starling Law of the beating heart and presumes that blood is ejected from the left ventricle into the aorta during chest compression.

Progressive decreases in left ventricular myocardial distensibility during chest compression contribute to progressive decline in the hemodynamic efficacy of closed-chest resuscitation. Studies in a porcine model of VF have shown that the severity of this phenomenon is proportional to the duration of untreated VF. (8)

In humans, Takino and Okada (13) reported on 59 adult patients who suffered non-traumatic out-of-hospital cardiac arrest and underwent open-chest direct manual cardiac compression in the emergency department after failure of closed-chest resuscitation. A "firm" myocardium was noticed during manual cardiac compression in 36 cases affecting predominantly the left ventricle. In the remaining 23 cases the hearts were "soft." They also noted that some hearts became "firm" during compression.

The presence of a "firm" myocardium was associated with reduced hemodynamic efficacy of cardiac compression as evidenced by a lower end-tidal $\mathrm{CO}_{2}$ tension $\left(\mathrm{P}_{\mathrm{ET}} \mathrm{CO}_{2}\right)$ - which is a well documented surrogate measurement of systemic and regional blood flow during cardiac resuscitation. (4,14-16) Hearts with "very firm" myocardium never regained spontaneous contractions. Hearts with "less firm" myocardium showed some, albeit insufficient, spontaneous contractions. Hearts with "soft" myocardium regained contractions and were able to generate a peripheral pulse in most instances.

Two lines of research support the feasibility of targeting mitochondrial bioenergetic function for resuscitation from cardiac arrest. One line relates to work using sodium-hydrogen exchanger isoform-1 (NHE-1) inhibitors in various animal models of cardiac arrest by our group over a period of approximately 10 years.n $(9,10,17-23)$ The other relates to more recent work using erythropoietin in a rat model of cardiac arrest (24) and in a small clinical study in patients suffering out-of-hospital cardiac arrest in collaboration with Dr. Štefek Grmec, MD, PhD and coworkers. (25) Both lines of research support the rationale and feasibility of using either an NHE-1 inhibitor or erythropoietin for preservation of left ventricular myocardial distensibility during cardiac resuscitation.

\section{NHE-1 Inhibitors}

The initial findings suggesting that NHE-1 inhibition could attenuate reductions in left ventricular myocardial distensibility during resuscitation and also prevent post-resuscitation diastolic dysfunction were made in an isolated (Langendorff) rat model of VF and simulated resuscitation $(17,18)$ establishing the rational for subsequent studies in intact rat and intact pig models of $V F$ and resuscitation. Findings in a pig model of VF and closed-chest resuscitation corroborated capability of NHE-1 inhibition to preserve left ventricular myocardial distensibility during closed-chest resuscitation evidenced by preservation of wall thickness and cavity size. Preservation of left ventricular myocardial distensibility enabled the generation of higher coronary perfusion pressures leading to higher resuscitability rates. (9)

Subsequent studies were conducted in an intact rat model of VF and closed-chest resuscitation designed to measure "using fluorescent microspheres" the effects of NHE-1 inhibition on systemic and organ blood flow as a function of compression depth. (21) We had reasoned that if left ventricular myocardial distensibility - and therefore preload - could be preserved by NHE-1 inhibition, then higher forward blood flows could be generated for a given compression depth; thus, shifting the relationship between flow and compression depth to the left. The studies indeed confirmed that NHE-1 inhibition allowed attaining comparable levels of systemic and organ blood flow with significantly less depth of chest compression. These studies also suggested that higher coronary perfusion pressures could be generated when administering a vasopressor agent given the larger blood flow generated for a given compression depth. This was the case when the NHE-1 inhibitor cariporide was combined with epinephrine in a pig model (22) and when combined with epinephrine or vasopressin in a rat model. (20)

An open-chest pig model of electricallyinduced VF and extracorporeal circula- 
tion was subsequently developed to study the myocardial energy effects of inhibiting NHE-1 under conditions of controlled coronary perfusion pressure. Myocardial tissue measurements indicated that NHE-1 inhibition preserved mitochondrial bioenergetic function during the interval of simulated resuscitation. This effect was supported by: i) higher creatine phosphate to creatine ratio, ii) higher ATP/ (Adenosine5'-diphosphate) ADP ratio, iii) lesser increases in adenosine, and iv) amelioration of myocardial lactate increases suggesting a shift away from anaerobic metabolism consequent to preservation of mitochondrial bioenergetic function.

\section{Erythropoietin}

Erythropoietin was studied in a rat model of VF and closed-chest resuscitation. (24) Erythropoietin given coincident with the beginning of chest compression after 10 minutes of untreated VF promoted hemodynamically more effective chest compression such that the coronary perfusion pressure to compression depth ratio averaged during the interval of chest compression was $25 \%$ higher than in control rats. Post-resuscitation, rats that received erythropoietin had less post-resuscitation myocardial dysfunction.

A subsequent clinical study in the city of Maribor, (25) Slovenia supported the potential benefits of erythropoietin for clinical resuscitation. Erythropoietin $(90,000$ IU of beta-epoetin) was given to 24 victims of out-of-hospital cardiac arrest and the effects compared prospectively with victims that received $0.9 \% \mathrm{NaCl}$ (concurrent controls $=30$ ) and retrospectively with a victims from a preceding interval treated with similar protocol (matched controls $=48$ ). By univariate analysis, administration of erythropoietin - when compared with concurrent controls - was associated with higher rates of intensive care unit (ICU) admission, return of spontaneous circulation (ROSC), 24-hour survival, and survival to hospital discharge and when compared with matched controls - it was associated with higher rates of ICU admission, ROSC, and 24-hour survival. After adjustment by pretreatment covariates, comparison with concurrent controls reduced the odds ratio but retained statistical significance for ICU admission and ROSC whereas comparison with matched controls increased the odds ratio demonstrating statistical significance for all four outcomes. To assess whether the beneficial effects on resuscitation outcomes could have been linked to beneficial effects on left ventricular myocardial distensibility - as suggested by our preceding study in rats (24) - we examine the effects on $\mathrm{P}_{E T} \mathrm{CO}_{2}$ "a good surrogate measurement of forward blood flow during chest compression" demonstrating that victims who received erythropoietin had significantly higher $\mathrm{P}_{\mathrm{ET}} \mathrm{CO}_{2}$ during chest compression.

Accordingly, these clinical observations - though based on a small sample size - were consistent with the hypothesis that erythropoietin - by preserving myocardial distensibility - leads to hemodynamically more effective chest compression resulting in higher resuscitation and survival rates.

\section{REFERENCES}

1. Binak K, Harmanci N, Sirmaci N, Ataman N, Ogan H. Oxygen extraction rate of the myocardium at rest and on exercise in various conditions. Br Heart J 1967;29:422-7.

2. Yusa T, Obara S. Myocardial oxygen extraction rate under general anesthesia. Tohoku J Exp Med 1981;133:321-4.

3. Hoffman JIE. Maximal coronary flow and the concept of coronary vascular reserve. Circulation 1984;70:153-9.

4. Duggal C, Weil MH, Gazmuri RJ, Tang W, Sun S, O'Connell F, Ali M. Regional blood flow during closed-chest cardiac resuscitation in rats. J Appl Physiol 1993;74:147-52.

5. Dong Z, Saikumar P, Weinberg JM, Venkatachalam MA. Calcium in cell injury and death. Annu Rev Pathol 2006;1:405-34.

6. Halestrap AP. Calcium, mitochondria and reperfusion injury: a pore way to die. Biochem Soc Trans 2006;34:232-7.

7. Weisfeldt ML, Zweier J, Ambrosio G, Becker LC, Flaherty JT. Evidence that free radicals result in reperfusion injury in heart muscle. Basic Life Sci 1988;49:911-9.

8. Klouche K, Weil MH, Sun S, Tang W, Povoas HP, Kamohara T, et al. Evolution of the stone heart after prolonged cardiac arrest. Chest 2002;122:1006-11.

9. Ayoub IM, Kolarova JD, Yi Z, Trevedi A, Deshmukh H, Lubell DL, Franz MR, Maldonado FA, Gazmuri RJ. Sodium-hydrogen exchange inhibition during ventricular fibrillation: Beneficial effects on ischemic contracture, action potential duration, reperfusion arrhythmias, myocardial function, and resuscitability. Circulation 2003;107:1804-9.

10. Ayoub IM, Kolarova J, Kantola R, Radhakrishnan J, Gazmuri RJ. Zoniporide preserves left ventricular compliance during ventricular fibrillation and minimizes post-resuscitation myocardial dysfunction through benefits on energy metabolism. Crit Care Med 2007;35:2329-36.

11. Gazmuri RJ. Effects of repetitive electrical shocks on postresuscitation myocardial function. Crit Care Med 2000;28:228-32.

12. Gazmuri RJ, Deshmukh S, Shah PR. Myocardial effects of repeated electrical defibrillations in the isolated fibrillating rat heart. Crit Care Med 2000;28:2690-6. 
13. Takino M, Okada Y. Firm myocardium in cardiopulmonary resuscitation. Resuscitation 1996;33:101-6.

14. Sanders AB, Atlas M, Ewy GA, Kern KB, Bragg S. Expired PCO2 as an index of coronary perfusion pressure. Am J Emerg Med 1985;3:147-9.

15. Gudipati CV, Weil MH, Bisera J, Deshmukh HG, Rackow EC. Expired carbon dioxide: A noninvasive monitor of cardiopulmonary resuscitation. Circulation 1988;77:234-9.

16. Rubertsson S, Karlsten R. Increased cortical cerebral blood flow with LUCAS; a new device for mechanical chest compressions compared to standard external compressions during experimental cardiopulmonary resuscitation. Resuscitation 2005;65:357-63.

17. Gazmuri RJ, Hoffner E, Kalcheim J, Ho H, Patel M, Ayoub IM, et al. Myocardial protection during ventricular fibrillation by reduction of proton-driven sarcolemmal sodium influx. J Lab Clin Med 2001;137:43-55.

18. Gazmuri RJ, Ayoub IM, Hoffner E, Kolarova JD. Successful ventricular defibrillation by the selective sodium-hydrogen exchanger isoform-1 inhibitor cariporide. Circulation 2001;104:234-9.

19. Gazmuri RJ, Ayoub IM, Kolarova JD, Karmazyn M. Myocardial protection during ventricular fibrillation by inhibition of the sodium-hydrogen exchanger isoform-1. Crit Care Med 2002;30:166-71.

20. Kolarova J, Yi Z, Ayoub IM, Gazmuri RJ. Cariporide potentiates the effects of epinephrine and vasopressin by nonvascular mechanisms during closed-chest resuscitation. Chest 2005;127:1327-34.

21. Kolarova JD, Ayoub IM, Gazmuri RJ. Cariporide enables hemodynamically more effective chest compression by leftward shift of its flowdepth relationship. Am J Physiol Heart Circ Physiol 2005;288:2904-11.

22. Ayoub IM, Kolarova J, Kantola RL, Sanders R, Gazmuri RJ. Cariporide minimizes adverse myocardial effects of epinephrine during resuscitation from ventricular fibrillation. Crit Care Med 2005;33:2599-605.

23. Wang S, Radhakrishnan J, Ayoub IM, Kolarova JD, Taglieri DM, Gazmuri RJ. Limiting sarcolemmal Na+ entry during resuscitation from VF prevents excess mitochondrial Ca2+ accumulation and attenuates myocardial injury. J Appl Physiol 2007;103:55-65.

24. Singh D, Kolarova JD, Wang S, Ayoub IM, Gazmuri RJ. Myocardial protection by erythropoietin during resuscitation from ventricular fibrillation. Am J Ther 2007;14:361-8.

25. Grmec S, Strnad M, Kupnik D, Sinkovic A, Gazmuri RJ. Erythropoietin facilitates the return of spontaneous circulation and survival in victims of out-of-hospital cardiac arrest. Resuscitation 2009;80:631-7. 